\title{
Numerical Simulation of the Countercurrent Flow in a Gas Centrifuge
}

L. D. Cloutman

R. A. Gentry

\section{DISCLAIMER}

This report was prepared as an scoonnt of work eponcored by an apoecy of the United States Governmont. Neither the Unived Ststes Government acr any acancy thoreof, nor any of their

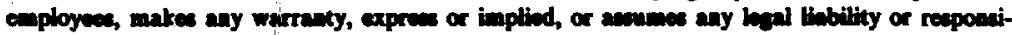

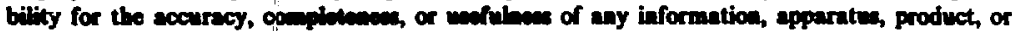
proceses diecloced, or represents thet its we would bot infriese privately owned rights. Referance herein to any apecific commercial product, proceas, or ervice by trade nane, trademark,

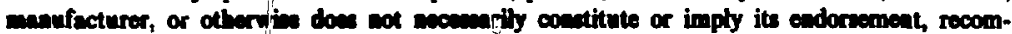
mendation, of favoring by the Unind Steve Goverament of any apacy thered. The viewe and opiniom of anthors exprened berein do not necenarily state or refloct those of the United States Goveranisen or any apeacy thoreof. 


\title{
MUMERICAL SIMULATION OF THE COUNTERCURREHT \\ FLOW IN A COS CENTRIFUGE
}

by

\author{
L. D. Cloutman and R. A. Gentry
}

ABSTRACT

We present a finite difference method for the numerical simulation of the axisymmetric countercurrent flow in a gas centrifuge. A timemarching technique is used to relax an arbitrary initial condition to the desired steady-state solution. All boundary layers can be resolved, and nonlinear effects may be included. Numerical examples are presented. We conclude that this technique is capable of predicting accurately the performance of a wide variety of machines under all operating conditions of interest.

\section{INTRODUCTION}

Our approach to solving the countercurrent problem is a partially implicit, finite difference, time-marching method applied to the linearized Navier-Stokes equations. In some applications nonlinearities are important, and some have been restored to the equations. Mass feed and scoop effects have been modeled by source terms as described by Wood and Sanders, ${ }^{1}$ but these will not be discussed in detail in this report. We obtain a solution by specifying an arbitrary initial condition, then marching through time until a steady state is reached. Separative work is then calculated by a method described by Park, ${ }^{2}$ which will not be discussed in this report.

Section II presents the governing equations. The numerical algorithm is presented in Sec. III, and two numerical examples are discussed in Sec. IV.

\section{GOVERNIMG EQUATIOWS}

Assume that the countercurrent in a gas centrifuge is adequately described by the equations of continuum fluid dynamics. The full nonlinear equations in cylindrical coordinates are 


$$
\begin{aligned}
& \frac{\partial \rho}{\partial t}+\frac{1}{r} \frac{\partial}{\partial r}(r u \rho)+\frac{\partial}{\partial z}(w \rho)=0 \\
& \rho \frac{\partial u}{\partial t}+\rho\left(u \frac{\partial u}{\partial r}+w \frac{\partial u}{\partial z}-\frac{v^{2}}{r}\right)=-\frac{\partial p}{\partial r}+2 \mu\left[\frac{\partial^{2} u}{\partial r^{2}}+\frac{1}{r}\left(\frac{\partial u}{\partial r}-\frac{u}{r}\right)\right] \\
& +\mu \frac{\partial}{\partial z}\left(\frac{\partial u}{\partial z}+\frac{\partial w}{\partial r}\right)+\lambda \frac{\partial}{\partial r}\left(\frac{1}{r} \frac{\partial r u}{\partial r}+\frac{\partial w}{\partial z}\right), \\
& \rho \frac{\partial v}{\partial t}+\rho\left(u \frac{\partial v}{\partial r}+w \frac{\partial v}{\partial z}+\frac{u v}{r}\right)=\mu\left[\frac{\partial^{2} v}{\partial z^{2}}+\frac{\partial^{2} v}{\partial r^{2}}-\frac{\partial}{\partial r}\left(\frac{v}{r}\right)+\frac{2}{r}\left(\frac{\partial v}{\partial r}-\frac{v}{r}\right)\right] \\
& \rho \frac{\partial w}{\partial t}+\rho\left(u \frac{\partial w}{\partial r}+w \frac{\partial w}{\partial z}\right)=-\frac{\partial p}{\partial z}+\mu\left[2 \frac{\partial^{2} w}{\partial z^{2}}+\frac{1}{r} \frac{\partial}{\partial r}\left(r \frac{\partial u}{\partial z}\right)+\frac{1}{r} \frac{\partial}{\partial r}\left(r \frac{\partial w}{\partial r}\right)\right] \\
& +\lambda \frac{\partial}{\partial z}\left(\frac{1}{r} \frac{\partial r u}{\partial r}+\frac{\partial w}{\partial z}\right),
\end{aligned}
$$

and

$$
\begin{aligned}
\rho \frac{\partial I}{\partial t} & +\rho\left(u \frac{\partial I}{\partial r}+w \frac{\partial I}{\partial z}\right)=-p\left(\frac{1}{r} \frac{\partial(r u)}{\partial r}+\frac{\partial w}{\partial z}\right)+\frac{\tau}{c_{v} r} \frac{\partial}{\partial r}\left(r \frac{\partial I}{\partial r}\right)+\frac{\tau}{c_{v}}-\frac{\partial^{2} I}{\partial z^{2}} \\
& +\lambda\left(\frac{1}{r} \frac{\partial(r u)}{\partial r}+\frac{\partial w}{\partial z}\right)^{2}+\mu\left[2\left(\frac{\partial u}{\partial r}\right)^{2}+2\left(\frac{\partial w}{\partial z}\right)^{2}+2\left(\frac{u}{r}\right)^{2}+\left(\frac{\partial v}{\partial z}\right)^{2}+\left(\frac{\partial u}{\partial z}\right)^{2}\right. \\
& \left.+\left(\frac{\partial w}{\partial r}\right)^{2}+2 \frac{\partial u}{\partial z} \frac{\partial w}{\partial r}+\left(\frac{\partial v}{\partial r}\right)^{2}-\frac{2 v}{r} \frac{\partial v}{\partial r}+\left(\frac{v}{r}\right)^{2}\right],
\end{aligned}
$$

where $\rho, u, v, w, I$, and $p$ are, respectively, the gas density, the radial, tangential, and axial velocity components, the specific internal energy and the pressure. We have assumed axial symmetry although all three velocity components are retained. The first and second viscosity coefficients and the thermal conductivity, $\mu, \lambda$, and $\tau$, respectively, are assumed to be constant. The ideal gas equation of state, 


$$
p=(\gamma-1) \rho I \quad
$$

is used in our calculations.

We linearize the flow equations by separating the solution into a zeroth order part and a perturbation term, and then keep only those terms linear in the perturbation quantities. For the zeroth order solution, denoted by the subscript 0 , we assume rigid body rotation and hydrostatic equilibrium in an isothermal fluid. Thèn

$$
\frac{\partial p_{0}}{\partial r}=\rho_{0} \omega^{2} r
$$

where $w$ is the angular velocity of the centrifuge. This can be integrated to give

$$
\begin{aligned}
p_{0} & =p_{\text {wall }} \exp \left[-A^{2}\left(1-\frac{r^{2}}{a^{2}}\right)\right] \\
& \equiv p_{\text {wall }} n,
\end{aligned}
$$

where

$$
A^{2}=\frac{a^{2} \omega^{2}}{2(\gamma-1) I_{0}} \equiv U^{2} / 2 .
$$

here, $\gamma$ is the ratio of specific heats and $a$ is the centrifuge rotor radius. From the equation of state and the isothermal assumption,

$$
\rho_{0}=p_{\text {wall }} \eta \text {. }
$$

The complete solution may be written

$$
\vec{u}=\vec{u}_{0}+\vec{u}^{\prime}=(0, w r, 0)+\left(u^{\prime}, v^{\prime}, w^{\prime}\right),
$$




$$
\begin{aligned}
& I=I_{0}+I^{\prime}, \\
& p=p_{0}+p^{\prime}=p_{\text {wall }} n+p^{\prime},
\end{aligned}
$$

and

$$
\rho=\rho_{0}+\rho^{\prime}=\rho_{\text {wall }}{ }^{n+\rho^{\prime}},
$$

where primes denote perturbations from the zeroth order solution. We linearize Eqs. (1) $-(5)$ by substituting in Eqs. (11)-(14) and dropping all terms that are quadratic in primed quantities. (Details are given by cloutman. ${ }^{3}$ ) The resulting 1 inear equations are

$$
\begin{aligned}
& \frac{\partial \rho^{\prime}}{\partial t}+\frac{1}{r} \frac{\partial}{\partial r}\left(r \rho_{0} u^{\prime}\right)+\frac{\partial}{\partial z}\left(\rho_{0} w^{\prime}\right)=0, \\
& \rho_{0} \frac{\partial u^{\prime}}{\partial t}=-\frac{\partial p^{\prime}}{\partial r}+\rho^{\prime} \omega^{2} r+2 \rho_{0} \omega v^{\prime}-\frac{\lambda u^{2}}{a^{2}} \frac{\partial}{\partial r}\left(r u^{\prime}\right)+\mu\left[\frac{1}{r} \frac{\partial}{\partial r}\left(r \frac{\partial u^{\prime}}{\partial r}\right)+\frac{\partial^{2} u^{\prime}}{\partial z^{2}}\right. \\
& \left.-\frac{u^{\prime}}{r^{2}}-\frac{u^{2}}{a^{2}} \frac{\partial}{\partial r}(r u l)\right] \\
& \rho_{0} \frac{\partial v^{\prime}}{\partial t}+2 \rho_{0} \text { wru' }=\mu\left[\frac{\partial^{2} v^{\prime}}{\partial z^{2}}+\frac{1}{r} \frac{\partial}{\partial r}\left(r \frac{\partial v^{\prime}}{\partial r}\right)-\frac{v^{\prime}}{r^{2}}\right] \text {, } \\
& \rho_{0} \frac{\partial w^{\prime}}{\partial t}=-\frac{\partial p^{\prime}}{\partial z}+\mu\left[\frac{\partial^{2} w^{\prime}}{\partial z^{2}}+\frac{1}{r} \frac{\partial}{\partial r}\left(r \frac{\partial w^{\prime}}{\partial r}\right)-\frac{u^{2} r}{a^{2}} \frac{\partial u^{\prime}}{\partial z}\right]-\frac{\lambda u^{2} r}{a^{2}} \frac{\partial u^{\prime}}{\partial z},
\end{aligned}
$$

and

$$
\rho_{0} \frac{\partial I^{\prime}}{\partial t}=-p_{0}\left(\frac{1}{r} \frac{\partial}{\partial r}\left(r u^{\prime}\right)+\frac{\partial w^{\prime}}{\partial z}\right)+\frac{\tau}{c_{v}}\left[\frac{1}{r} \frac{\partial}{\partial r}\left(r \frac{\partial I^{\prime}}{\partial r}\right)+\frac{\partial^{2} I^{\prime}}{\partial z^{2}}\right] \text {. }
$$


We transform our equations to dimensionless form using the following definitions:

$$
\begin{aligned}
& \left(u^{\prime}, v^{\prime}, w^{\prime}\right)=w a(\delta u, \delta v, \delta w), \\
& (r, z)=a\left(r^{\prime}, z^{\prime}\right), \\
& T^{\prime}=T_{0} \delta \theta, \\
& p^{\prime}=p_{0} \delta p, \\
& w t=t^{\prime}, \\
& B=1+\lambda / \mu,
\end{aligned}
$$

and

$$
\operatorname{Re}=\rho_{\text {wall }} \omega \mathrm{a}^{2} / \mu .
$$

The resulting equations are

$$
\begin{aligned}
\frac{\partial \delta p}{\partial t^{\prime}}-\frac{\partial \delta \theta}{\partial t^{\prime}}+\frac{1}{r^{\prime}} \eta \frac{\partial}{\partial r^{\prime}}\left(r^{\prime} n \delta u\right)+\frac{\partial}{\partial z^{\prime}} \delta w=0, & \\
\frac{\partial \delta u}{\partial t^{\prime}} & =-\frac{1}{u^{2}} \frac{\partial \delta p}{\partial r^{\prime}}-r^{\prime} \delta \theta+2 \delta v-\frac{B u^{2}}{R e n} \frac{\partial}{\partial r^{\prime}}\left(r^{\prime} \delta u\right)+\frac{1}{R e n}\left[\frac{1}{r^{\prime}} \frac{\partial}{\partial r^{\prime}}\left(r^{\prime} \frac{\partial \delta u}{\partial r^{\prime}}\right)\right. \\
& \left.+\frac{\partial^{2} \delta u}{\partial z^{\prime 2}}-\frac{\delta u}{r^{\prime 2}}\right], \\
\frac{\partial \delta v}{\partial t^{\prime}} & =-2 \delta u+\frac{1}{R e} n\left[\frac{1}{r^{\prime}} \frac{\partial}{\partial r^{\prime}}\left(r^{\prime} \frac{\partial \delta v}{\partial r^{\prime}}\right)+\frac{\partial^{2} \delta v}{\partial z^{\prime 2}}-\frac{\delta v}{r^{\prime 2}}\right],
\end{aligned}
$$




$$
\frac{\partial \delta w}{\partial t^{\top}}=-\frac{1}{U^{2}} \frac{\partial \delta p}{\partial z^{\prime}}-\frac{B U^{2}}{R e n} r^{\prime} \frac{\partial \delta U}{\partial z^{\prime}}+\frac{1}{R e n}\left[\frac{1}{r^{\prime}} \frac{\partial}{\partial r^{\prime}}\left(r^{\prime} \frac{\partial \delta w}{\partial r^{\prime}}\right)+\frac{\partial^{2} \delta w}{\partial z^{\prime 2}}\right] \text {, }
$$

and

$$
\frac{\partial \delta \theta}{\partial t^{\prime}}=(r-1) U^{2} r^{\prime} \delta u+\frac{\gamma}{\operatorname{Re} \operatorname{Pr} \eta}\left[\frac{1}{r^{\prime}} \frac{\partial}{\partial r^{\prime}}\left(r^{\prime} \frac{\partial \delta \theta}{\partial r^{\prime}}\right)+\frac{\partial^{2} \delta \theta}{\partial z^{\prime 2}}\right],
$$

where the Prandtl number $\operatorname{Pr}$ is defined as $\operatorname{Pr}=c_{p} \nu / \tau$.

We have retained all of the linear terms. For certain problems, we must also include some of the nonlinear terms. Order of magnitude analys is suggests that PdV work, the Coriolis force, and convection of internal energy and angular momentum are the most important nonlinear terms. By dropping the primes and de?tas to make the notation less cumbersome, we obtain the final scaled equations

$$
\begin{aligned}
& \frac{\partial p}{\partial t}-\frac{\partial \theta}{\partial t}+\frac{1}{r \eta} \cdot \frac{\partial}{\partial r}(r n u)+\frac{\partial w}{\partial z}=q_{\rho}, \\
& \frac{\partial u}{\partial t}=-\frac{1}{u^{2}} \frac{\partial p}{\partial r}-r \theta+2 v-\frac{B u^{2}}{\operatorname{Re} \eta} \frac{\partial}{\partial r}(r u)+\frac{1}{\operatorname{Re} \eta}\left[\frac{1}{r} \frac{\partial}{\partial r}\left(r \frac{\partial u}{\partial r}\right)+\frac{\partial^{2} u}{\partial z^{2}}-\frac{u}{r^{2}}\right]+q_{u} \text {, } \\
& \frac{\partial v}{\partial t}+\frac{1}{n r} \frac{\partial}{\partial r}(r n v u)+\frac{\partial}{\partial z}(v w)=-2 u-\frac{u v}{r}+\frac{1}{\operatorname{Re} \eta}\left[\frac{1}{r} \frac{\partial}{\partial r}\left(r \frac{\partial v}{\partial r}\right)+\frac{\partial^{2} v}{\partial z^{2}}-\frac{v}{r^{2}}\right]+q v, \\
& \frac{\partial w}{\partial t}=-\frac{1}{U^{2}} \frac{\partial p}{\partial z}-\frac{B U^{2}}{\operatorname{Re} \eta} r \frac{\partial u}{\partial z}+\frac{1}{\operatorname{Re} \eta}\left[\frac{1}{r} \frac{\partial}{\partial r}\left(r \frac{\partial w}{\partial r}\right)+\frac{\partial^{2} w}{\partial z^{2}}\right]+q_{w},
\end{aligned}
$$

and 


$$
\begin{aligned}
\frac{\partial \theta}{\partial t} & +\frac{1}{r n} \frac{\partial}{\partial r}(r n \theta u)+\frac{\partial}{\partial z}(\theta w)=-(\gamma-1)(1+p)\left[\frac{1}{r} \frac{\partial}{\partial r}(r u)+\frac{\partial w}{\partial z}\right] \\
& +\frac{\gamma}{\operatorname{Re} \operatorname{Pr} \eta}\left[\frac{1}{r} \frac{\partial}{\partial r}\left(r \frac{\partial \theta}{\partial r}\right)+\frac{\partial^{2} \theta}{\partial z^{2}}\right]+q_{I} .
\end{aligned}
$$

The scaled source terms in the rotating reference frame, $q_{\rho}$, etc., are introduced to allow calculation of solutions that include the effects of feed and scoops. These functions are constant in time and were introduced by the authors in some earlier unpublished work. Wood and Sanders ${ }^{1}$ subsequently described this procedure as adapted to their pancake model. We include them for completeness although they are not used in the present numerical examples.

\section{THE MUMERICAL METHOD}

Two features of the centrifuge countercurrent problem increase the difficulty of obtaining numerical solutions. First, the density can vary by a large factor across the radius of the machine. This forces the use of implicit diffusion terns to make the scheme efficient.

The second feature of the problem is the importance of the thin boundary layers on the endcaps and rotor wall. Our algorithm employs a nonuniform grid that allows resolution of all boundary layers. Computational cells are required to be rectangular, but the widths can be varied to obtain sufficient resolution next to the rotor wall and endcaps. The small cells in the boundary layers also require the use of implicit diffusion terms to avoid unacceptably small time steps. For computational convenience in applying boundary conditions, a single row of "fictitious" cells surrounds the grid.

The radial and axial cell edge locations $r_{j-\frac{1}{2}}$ and $z_{j-\frac{1}{2}}$ are specified by the user. The centers of our rectangular cells are defined by $\left(r_{i}, z_{j}\right)=\left(0.5\left(r_{i-\frac{1}{2}}+\right.\right.$ $\left.\left.r_{i+\frac{1}{2}}\right), 0.5\left(z_{j-\frac{1}{2}}+z_{j+\frac{1}{2}}\right)\right)$. It is also necessary to distinguish between the cell edge positions, for example $r_{j-\frac{1}{2}}$, and the average position of two adjacent cell centers, for example $\bar{r}_{\mathbf{i}-\frac{1}{2}}=0.5\left(r_{\mathbf{i}}+r_{j-1}\right)$. The variables $\theta, p$, and $v$ are defined at the cell centers. The velocity components $u$ and $w$ are defined on the left and bottom cell edges respectively.

It is convenient (and efficient in the computer program) to define a series of coefficients that represent the mesh-dependent parts of the various finite difference operators. Let 


$$
\begin{aligned}
& B_{i}^{1}=\frac{\bar{r}_{i+\frac{1}{2}}}{r_{i}\left(\bar{r}_{i+\frac{1}{2}}-\bar{r}_{i-\frac{1}{2}}\right)\left(r_{i+1}-r_{1}\right)}, \\
& B_{i}^{2}=\frac{\bar{r}_{j-\frac{1}{2}}}{r_{j}\left(\bar{r}_{j+\frac{1}{2}}-\bar{r}_{j-\frac{1}{2}}\right)\left(r_{i}-r_{j-1}\right)} \text {, } \\
& B_{j}^{3}=1 /\left(\bar{z}_{j+\frac{1}{2}}-\bar{z}_{j-\frac{1}{2}}\right)\left(z_{j+1}-z_{j}\right), \\
& B_{j}^{4}=1 /\left(\bar{z}_{j+\frac{1}{2}}-\bar{z}_{j-\frac{1}{2}}\right)\left(z_{j}-z_{j-1}\right), \\
& \mathrm{B}_{i}^{20}=\mathrm{B}_{i}^{1}+\mathrm{B}_{i}^{2}+\frac{1}{r_{i}^{2}} \text {, } \\
& B_{j}^{21}=B_{j}^{3}+B_{j}^{4}, \\
& B_{i}^{22}=B_{i}^{1}+B_{i}^{2}, \\
& B_{j-\frac{1}{2}}^{7}=1 /\left(z_{j}-z_{j-1}\right)\left(z_{j+\frac{1}{2}}-z_{j-\frac{1}{2}}\right), \\
& B_{j-\frac{1}{2}}^{8}=1 /\left(z_{j}-z_{j-1}\right)\left(z_{j-\frac{1}{2}}-z_{j-\frac{3}{2}}\right) \\
& B_{j-\frac{1}{2}}^{23}=1 / U^{2}\left(z_{j}-z_{j-1}\right), \\
& B_{j-\frac{1}{2}}^{24}=A^{2} B /\left(z_{j}-z_{j-1}\right), \\
& B_{i}^{25}=r_{i} / \operatorname{Re} n_{i},
\end{aligned}
$$




$$
\begin{aligned}
& B_{j-\frac{1}{2}}^{26}=B_{j-\frac{1}{2}}^{7}+B_{j-\frac{1}{2}}^{8}, \\
& B_{i-\frac{1}{2}}^{32}=B U^{2}+B_{i-\frac{1}{2}}^{5}+B_{i-\frac{1}{2}}^{6}+\frac{1}{r_{i-\frac{1}{2}}^{2}}, \\
& B_{i-\frac{1}{2}}^{27}=1 / N^{2}\left(r_{i}-r_{i-1}\right), \\
& B_{i-\frac{1}{2}}^{34}=B U^{2} r_{i-\frac{1}{2}} /\left[\operatorname{Re} \eta_{i-\frac{1}{2}}\left(r_{i+\frac{1}{2}}-r_{i-\frac{3}{2}}\right)\right], \\
& B_{i-\frac{1}{2}}^{5}=r_{j} / r_{i-\frac{1}{2}}\left(r_{j}-r_{j-1}\right)\left(r_{i+\frac{1}{2}}-r_{j-\frac{1}{2}}\right), \\
& B_{i-\frac{1}{2}}^{6}=r_{i-1} / r_{i-\frac{1}{2}}\left(r_{i}-r_{i-1}\right)\left(r_{i-\frac{1}{2}}-r_{i-\frac{3}{2}}\right), \\
& B_{i}^{9}=\frac{r_{i+\frac{1}{2}} n_{i+\frac{1}{2}}}{n_{i} r_{j}\left(r_{i+\frac{1}{2}}-r_{i-\frac{1}{2}}\right)} \text {, } \\
& B_{i}^{10}=\frac{r_{j-\frac{1}{2}} n_{i-\frac{1}{2}}}{n_{i} r_{j}\left(r_{j+\frac{1}{2}}-r_{j-\frac{1}{2}}\right)}, \\
& B_{i}^{11}=\frac{r_{j+\frac{1}{2}} n_{i+\frac{1}{2}} B_{j+\frac{1}{2}}^{27}}{n_{j} r_{j}\left(r_{j+\frac{1}{2}}-r_{j-\frac{1}{2}}\right)}, \\
& B_{i}^{12}=\frac{r_{i-\frac{1}{2}}{ }^{\eta_{i-\frac{1}{2}}} B_{i-\frac{1}{2}}^{27}}{\eta_{i} r_{j}\left(r_{j+\frac{1}{2}}-r_{i-\frac{1}{2}}\right)}, \\
& B_{j}^{13}=\frac{B_{j+\frac{1}{2}}^{23}}{z_{j+\frac{1}{2}}-z_{j-\frac{1}{2}}},
\end{aligned}
$$

and

9 
$B_{j}^{14}=\frac{B_{j-\frac{1}{2}}^{23}}{z_{j+\frac{1}{2}}-z_{j-\frac{1}{2}}}$.

The difference equations for the advanced time (denoted by a superscript

$n+1)$ radial and axial velocities are

$$
\begin{aligned}
u_{i-\frac{1}{2} j}^{n+1}[1 & \left.+\frac{\delta t}{\operatorname{Re} n_{i-\frac{1}{2}}}\left(B_{i-\frac{1}{2}}^{32}+B_{j}^{21}\right)\right]=u_{i-\frac{1}{2} j}^{n}+\delta t\left\{B_{i-\frac{1}{2}}^{27}\left(p_{i-1 j}^{n+1}-p_{i j}^{n+1}\right)+v_{i j}^{n}\right. \\
& +v_{i-1 j}^{n}-\frac{r_{i-\frac{1}{2}}}{2}\left(\theta_{i j}^{n}+\theta_{i-1 j}^{n}\right)-B_{i-\frac{1}{2}}^{34}\left(u_{i+\frac{1}{2} j}^{n}-u_{i-\frac{3}{2} j}^{n}\right)+\left(a_{u}\right)_{i-\frac{1}{2} j} \\
& \left.+\frac{1}{\operatorname{Re} n_{i-\frac{1}{2}}}\left[B_{i-\frac{1}{2}}^{5} u_{i+\frac{1}{2} j}^{n}+B_{i-\frac{1}{2}}^{6} u_{i-\frac{3}{2} j}^{n}+B_{j}^{3} u_{i-\frac{1}{2} j+1}^{n}+B_{j}^{4} u_{i-\frac{1}{2} j-1}^{n}\right]\right\}
\end{aligned}
$$

and

$$
\begin{aligned}
w_{i j-\frac{1}{2}}^{n+1}[1 & \left.+\frac{\delta t}{\operatorname{Re} n_{j}}\left(B_{i}^{22}+B_{j-\frac{1}{2}}^{26}\right)\right]=w_{i j-\frac{1}{2}}^{n}+\delta t\left\{B_{j-\frac{1}{2}}^{23}\left(p_{i j-1}^{n+1}-p_{i j}^{n+1}\right)\right. \\
& -B_{j-\frac{1}{2}}^{24} B_{i}^{25}\left(u_{i+\frac{1}{2} j}^{n}+u_{i-\frac{1}{2} j}^{n}-u_{i+\frac{1}{2} j-1}^{n}-u_{i-\frac{1}{2} j-1}^{n}\right)+\left(q_{w}\right)_{i j-\frac{1}{2}} \\
& +\frac{1}{\operatorname{Re} \eta_{i}}\left(B_{i}^{1} w_{i+1 j-\frac{1}{2}}^{n}+B_{i}^{2} w_{i-1 j-\frac{1}{2}}^{n}+B_{j-\frac{1}{2}}^{7} w_{i j+\frac{1}{2}}^{n}+B_{j-\frac{1}{2}}^{8} w_{i j-\frac{3}{2}}^{n}\right\} .
\end{aligned}
$$

Next, define $\alpha, \Omega, \alpha$, and $\Sigma$ by writing Eqs. (61) and (62) as

$$
\begin{aligned}
& u_{i-\frac{1}{2} j}^{n+1}\left[1+\frac{\delta t}{\operatorname{Re} n_{i-\frac{1}{2}}}\left(B_{i-\frac{1}{2}}^{32}+B_{j}^{21}\right)\right] \equiv u_{i-\frac{1}{2} j}^{n+1} / \sigma_{i-\frac{1}{2} j} \\
& =\sum_{i-\frac{1}{2} j}+\delta t B_{i-\frac{1}{2}}^{27}\left(p_{i-1 j}^{n+1}-p_{i j}^{n+1}\right)
\end{aligned}
$$


and

$$
\begin{gathered}
w_{i j-\frac{1}{2}}^{n+1}\left[1+\frac{1}{\operatorname{Re} \eta_{i}}\left(B_{i}^{22}+B_{j-\frac{1}{2}}^{26}\right)\right] \equiv w_{i j-\frac{1}{2}}^{n+1} / \alpha_{i j-\frac{1}{2}} \\
=\Omega_{i j-\frac{1}{2}}+\delta t B_{j-\frac{1}{2}}^{23}\left(p_{i j-1}^{n+1}-p_{i j}^{n+1}\right) .
\end{gathered}
$$

The first step in each cycle is to evaluate $\alpha, \Omega, \sigma$, and $\Sigma$ for each cell face. Next we convicrt. Eq. (32) into a Poisson equation for the advanced time pressure. First, difference Eq. (32) to obtain

$$
\begin{gathered}
\frac{p_{i j}^{n+1}-p_{i j}^{n}}{\delta t}-\frac{\theta_{i j}^{n+1}-\theta_{i j}^{n}}{\delta t}+\frac{1}{r_{i} n_{j}\left(r_{i+\frac{1}{2}}-r_{i-\frac{1}{2}}\right)}\left(r_{i+\frac{1}{2}} n_{i+\frac{1}{2}} u_{i+\frac{1}{2} j}^{n+1}\right. \\
\left.-r_{i-\frac{1}{2}} n_{i-\frac{1}{2}} u_{i-\frac{1}{2} j}^{n+1}\right)+\frac{w_{i j+\frac{1}{2}}^{n+1}-w_{i j-\frac{1}{2}}^{n+1}}{\left(z_{j+\frac{1}{2}}-z_{j-\frac{1}{2}}\right.}=\left(q_{\rho}\right)_{i j} .
\end{gathered}
$$

Let $Q_{i j}$ be defined by

$$
\begin{aligned}
Q_{i j} & =p_{i j}^{n}+\theta_{i j}^{n+1}-\theta_{i j}^{n}+\delta t\left\{B_{i}^{10} \sigma_{i-\frac{1}{2} j} \sum_{j-\frac{1}{2} j}-B_{j}^{9} \sigma_{i+\frac{1}{2} j} \sum_{j+\frac{1}{2} j}\right. \\
& \left.+\frac{1}{z_{j+\frac{1}{2}}-z_{j-\frac{1}{2}}}\left(\alpha_{i j-\frac{1}{2}} \Omega_{i j-\frac{1}{2}}-\alpha_{i j+\frac{1}{2}} \Omega_{i j+\frac{1}{2}}\right)+\left(q_{p}\right)_{i j}\right\} .
\end{aligned}
$$

By substituting Eqs. (63) and (64) into (65) and by simplifying with the aid of Eq. (66), we obtain

$$
\begin{aligned}
p_{i j}^{n+1}[1 & \left.+\delta t^{2}\left(B_{i}^{11} \sigma_{i+\frac{1}{2} j}+B_{i}^{12} \sigma_{i-\frac{1}{2} j}+B_{j}^{13} \alpha_{i j+\frac{1}{2}}+B_{j}^{14} \alpha_{i j-\frac{1}{2}}\right)\right]=Q_{i j} \\
& +\delta t^{2}\left(B_{i}^{11} \sigma_{i+\frac{1}{2} j} p_{i+1 j}^{n+1}+B_{i}^{12} \sigma_{i-\frac{1}{j} j} p_{i-1 j}^{n+1}+B_{j}^{13} \alpha_{i j+\frac{1}{2}} p_{i j+1}^{n+1}\right. \\
& \left.+B_{j}^{14} \alpha_{i j-\frac{1}{2}} p_{i j-1}^{n+1}\right) .
\end{aligned}
$$


The boundary condition is zero pressure gradient normal to each boundary. This is accomplished in the code by requiring the pressure in each fictitious cell just outside the mesh boundary to be equal to the pressure just inside. We also require $\Sigma_{i-\frac{1}{2} j}=\Omega_{i j-\frac{1}{2}}=0$ and $\sigma_{j-\frac{1}{2} j}=\alpha_{i j-\frac{1}{2}}=1$ on the boundaries. Eq. (67) may be solved by any standard iterative technique, such as successive overrelaxation (SOR).

Although this approach is computationally efficient for a single computational cycle, the solution usually contains slowly-damped waves initiated by the initial conditions. These waves may be eliminated by setting the time derivatives of $\theta$ and $p$ to zero in Eq. (65), which requires deletion of the first three terins on the right-hand side of Eq. (66) and deletion of the 1 from the quantity in square brackets in Eq. $(67)$.

We have been unable to find a suitable iterative scheme for solving the modified pressure equation, so we use a special linear system solver written specifically for this sparse, five-striped matrix. ${ }^{4}$ This scheme uses LU decomposition, and the $L^{\prime \prime}$ and $U$ factors are saved from cycle to cycle when is is constant, which greatly improves efficiency. The zero normal gradient condition is coded into the coefficient natrix so the solution automatically satisfies boundary conditions.

This modified pressure equation has an infinite number of sclutions, each differing by an additive constant. For linear calculations, only $\nabla p$ enters the calculation and the value of the constant is irrelevant. For nonlinear calculations, the magnitude of $p$ enters the PdV work term and the constant is not arbitrary. The linear system solver finds one particular member of the family of solutions, $\bar{p}_{i j}$. The normalization constant is chosen so that the integral of the density perturbation over the volume of the rotor vanishes. In scaled finite difference form, this condition is

$$
\sum_{i} \sum_{j}\left(\bar{p}_{i j}+h-\theta_{i j}\right) n_{i} r_{i}\left(r_{i+\frac{1}{2}}-r_{i-\frac{1}{2}}\right)\left(z_{j+\frac{1}{2}}-z_{j-\frac{1}{2}}\right)=0,
$$

where the sums are over all real cells. Eq. (68) is solved for $h$, and

$$
p_{i j}^{n+1}=\bar{p}_{i j}+n \text {. }
$$


This procedure requires more time per cycle than the iterative, fully timedependent scheme, but fewer cycles are required to relax a solution to steady state. Computational times for a given problem are approximately the same for the two methods. Separation theory requires that $\nabla \cdot\left(\rho_{0} u\right)=0$ to a high degree of accuracy. The matrix solution satisfies this condition to within computer roundoff on every cycle, which makes the matrix approach preferable to the iterated method if computer menory does not seriously limit the size of $L$ and $U$ factors that may be stored.

The next step in the computational cycle is to calculate $u_{i-\frac{1}{2} j}^{n+1}$ and $w_{i j-\frac{1}{2}}^{n+1}$ from Eqs. (63) and (64).

The angular monentum equation is solved next. The finite difference approximation is

$$
\begin{aligned}
v_{i j}^{n+1}[1 & \left.+\frac{\delta t}{\operatorname{Re} n_{i}}\left(B_{i}^{20}+B_{j}^{21}\right)\right]=v_{i j}^{n}+\delta t\left\{-u_{i-\frac{1}{2} j}^{n+1}-u_{i+\frac{1}{2} j}^{n+1}\right. \\
& -\frac{\xi\left(u_{i-\frac{1}{2} j}^{n+1}+u_{i+\frac{1}{2} j}^{n+1}\right) v_{i j}^{n+1}}{2 r_{i}}+\left(q_{v}\right)_{i j}-\frac{\xi}{2}\left[B_{i}^{9} u_{i+\frac{1}{2} j}^{n+1}\left(v_{i+1 j}^{n+1}+v_{i j}^{n+1}\right)\right. \\
& -B_{i}^{10} u_{i-\frac{1}{2} j}^{n+1}\left(v_{i j}^{n+1}+v_{i-1 j}^{n+1}\right) \\
& \left.+\frac{\left.w_{i j+\frac{1}{2}}^{n+1}\left(v_{i j+1}^{n+1}+v_{i j}^{n+1}\right)-w_{i j-\frac{1}{2}}^{n+1}\left(v_{i j}^{n+1}+v_{i j-1}^{n+1}\right)\right]}{z_{j+\frac{1}{2}}-z_{j-\frac{1}{2}}}\right] \\
& \left.+\frac{1}{\operatorname{Re} n_{j}}\left[B_{i}^{1} v_{i+1 j}^{n+1}+B_{i}^{2} v_{i-1 j}^{n+1}+B_{j}^{3} v_{i j+1}^{n+1}+B_{j}^{4} v_{i j-1}^{n+1}\right]\right\}
\end{aligned}
$$

This equation is fully implicit in $\checkmark$ and it is readily solved by SOR. Similarly, we solve

$$
\begin{aligned}
\theta_{i j}^{n+1}[1 & \left.+\frac{\gamma \delta t}{\operatorname{Re} \operatorname{Pr} n_{i}}\left(B_{j}^{21}+B_{i}^{22}\right)\right]=\theta_{i j}^{n}+\delta t\left\{( \gamma - 1 ) A ^ { 2 } r _ { i } \left(u_{i+\frac{1}{2} j}^{n+1}\right.\right. \\
& \left.+u_{i-\frac{1}{2}}^{n+1}\right)(1-\xi)+\xi(\gamma-1)\left(1+p_{i j}^{n+1}\right)\left[\left(r_{i+\frac{1}{2}} u_{i+\frac{1}{2} j}^{n+1}-r_{i-\frac{1}{2}} u_{i-\frac{1}{2} j}^{n+1}\right) / r_{j}\left(r_{i+\frac{1}{2}}\right.\right.
\end{aligned}
$$




$$
\begin{aligned}
& \left.\left.-r_{i-\frac{1}{2}}\right)+\left(w_{i j+\frac{1}{2}}^{n+1}-w_{i j-\frac{1}{2}}^{n+1}\right) /\left(z_{j+\frac{1}{2}}-z_{j-\frac{1}{2}}\right)\right]+\left(a_{I}\right)_{i j} \\
& -\frac{\xi}{2}\left[B_{i}^{9} u_{i+\frac{1}{2} j}^{n+1}\left(\theta_{i+1 j}^{n+1}+\theta_{i j}^{n+1}\right)-B_{i}^{10} u_{i-\frac{1}{2} j}^{n+1}\left(\theta_{i j}^{n+1}+\theta_{i-1 j}^{n+1}\right)\right. \\
& \left.+\frac{w_{i j+\frac{1}{2}}^{n+1}\left(\theta_{i j+1}^{n+1}+\theta_{i j}^{n+1}\right)-w_{i j-\frac{1}{2}}^{n+1}\left(\theta_{i j}^{n+1}+\theta_{i j-1}^{n+1}\right)}{z_{j+\frac{1}{2}}^{n+z_{j-\frac{1}{2}}}}\right] . \\
& \left.+\frac{\gamma}{\operatorname{Re} \operatorname{Pr} n_{i}}\left[B_{i}^{1} \theta_{i+1 j}^{n+1}+B_{i}^{2} \theta_{i-1 j}^{n+1}+B_{j}^{3} \theta_{i j+1}^{n+1}+B_{j}^{4} \theta_{i j-1}^{n+1}\right]\right\} .
\end{aligned}
$$

by SOR for the advanced time temperature. The parameter $\xi$ is zero or unity for linear and nonlinear solutions respectively. This completes a computational cycle.

The boundary conditions on the variables other than $p$ depend on the problem being solved, and they are straightforward to implement. As an example, we give the boundary conditions for a thermally driven solution, which is one of our numerical examples in tive next section.

(1) Left edge:

$$
\frac{\partial w}{\partial r}=\frac{\partial v}{\partial r}=\frac{\partial \theta}{\partial r}=u=0
$$

(2) Rotor wal1:

$$
w=v=u=0
$$

and

$$
\theta=\theta_{0}(L-z) / L,
$$

where $\theta_{0}$ is a constant and $L$ is the machine half-length in radii. 

(3) Bottom endcap $(z=0)$ :
$u=v=w=0$

and

$$
\theta=\theta_{0}
$$

(4) Top endcap $(z=2 L)$ :

$u=v=w=0$

and

$$
\theta=-\theta_{0}
$$

Now we discuss how the boundary conditions are applied numerically. Three examples serve to explain all types of numerical boundary conditions.

(1) $u=0$ on rotor wa11: Since $u$ is defined on the wal1, it is kept at that value for the entire calculation.

(2) $\frac{\partial \theta}{\partial r}=0$ on the left edge of the mesh: This condition is imposed by $\theta_{1 j}=\theta_{2 j}$.

(3) $\theta= \pm e_{0}$ on the endcaps.

Consider the bottom endcap. Traditionally, this boundary condition is handled by setting

$$
\theta_{i 1}=2 \theta_{0}-\theta_{i 2}
$$

However, this definition of $\theta_{i 1}$ gives (for a uniform mesh)

$$
\frac{{ }^{\theta} i 3-2 \theta i 2+\theta_{11}}{\delta z^{2}}=\left.\frac{3}{4} \frac{\partial^{2} \theta}{\partial z^{2}}\right|_{z_{2}}+o(\delta z),
$$


which has à large zeroth order truncation error. We use a scheme proposed by Buzbee* to eliminate the zeroth order error. We define $\theta_{i 1}$ so that we get the correct zeroth order value for $\frac{\partial^{2} \theta}{\partial z^{2}}$ at cell center i2:

$$
\frac{1}{\delta z^{2}}\left[\theta_{i 3}-2 \theta_{i 2}+\theta_{i 1}\right]=\left(\frac{\theta_{i 3}-\theta_{0}}{1.5 \delta z}-\frac{\theta_{i 2}-\theta_{0}}{0.5 \delta z}\right) \frac{1}{0.5 \delta z} .
$$

By solving for $\theta_{i 1}$, we obtain

$$
\theta_{i 1}=\frac{8}{3} \theta_{0}-2 \theta_{i 2}+\frac{1}{3} \theta_{i 3}
$$

The other boundary conditions may all be treated explicitly, but Eq. (82) must be done semi-implicitly for purposes of numerical stability. The coefficients of Eq. (82) are also correct for evaluating the fictitious cell quantities for $\frac{1}{r} \frac{\partial}{\partial r}\left(r \frac{\partial \theta}{\partial r}\right)$.

\section{MUMERICAL EXAMPLES}

The two numerical examples that we present in this section are the thermally driven and mass driven $\mathrm{SF}_{6}$ machines described by Nakayama and Usui. ${ }^{5}$ Our calculations were performed with the linear version of the program using the parameters listed below.

$$
\begin{aligned}
a & =10 \mathrm{~cm} \\
2 L a & =80 \mathrm{~cm} \\
A^{2} & =1.855 \\
R e & =1.94 \times 10^{6} \\
r & =1.0935 \\
P r & =0.7 \\
T_{0} & =320 \mathrm{~K} \\
w a & =260 \mathrm{~m}-\mathrm{s}^{-1} \\
\rho_{w} & =1.097 \times 10^{-3} \mathrm{~g} \mathrm{~cm}^{-3} \\
P_{w} & =150 \mathrm{torr} \\
\mu & =1.47 \times 10^{-4} \mathrm{P}^{-3}
\end{aligned}
$$

${ }^{\star}$ Information provided by B. L. Buzbee, Los Alamos Scientific Laboratory (1973). 
The first case is the thermally driven solution with a $10 \mathrm{~K}$ end-to-end temperature difference. The endcaps have a constant temperature $\theta_{0}= \pm 5 \mathrm{~K}$, and the $\theta$ profile on the rotor wall is linear. The calculation was performed on two computational grids. Case $A$ used a grid that filled the entire rotor in both directions; that is, $0<r<1$ and $0<z<2$ L. The radial grid was almost uniform in the ininer $30 \%$ of the machine, with or $\approx 0.05$. The cells were smoothly reduced in size at progressively larger radi until $\delta r=1.56 \times 10^{-3}$ at the $r$ tor wall. The axial grid began with $\delta z=1.53 \times 10^{-4}$ at the bottom endcap. The cell size was allowed to grow by a factor of approximately 1.4 for each succeeding cell until $\delta z=0.222$ for about 15 cells covering about $80 \%$ of the length of the rotor. The cells were gradualiy reduced in size until $\delta z=1.53 \times 10^{-4}$ at the top endcap. This grid was 32 cells by 78 cells. It allowed us to resolve all three boundary layers. There were eight cells in the downflow next to the rotor wall and three to four cells in the Ekman layers on the endcaps.

The inner $80 \%$ of the machine was essentially inert, so we ran case $B$ with the inner boundary at $r=0.7$ instead of $r=0$. The sane number zones and the same axial grid were used. Resolution was improved in the radial direction, with $\delta r=0.012$ at the inner boundary and $\delta r=1.50 \times 10^{-3}$ at the rotor wall. The results are almost indistinguishable from Case $A$.

Figure 1 shows streamlines for Case $B$, which may be compared to the top half: of Fig. 3 of Nakayama and Usui. Both their solution and ours are symnetric about the rotor midplane. The radial locations of the centers of vorticity and the dividing streamline between the outer pair of vortices agree well. No difference can be seen within the accuracy to which the figures can be read. The contours were chosen to be the same as those presented by Nakayama and Usui to the extent possible wher using existing computer-generated streamline plots. The 0,10, and $20 \mathrm{mg} / \mathrm{s}$ curves may be compared directly. In particular, the $20 \mathrm{mg} / \mathrm{s}$ curves in both solution; occupy almost exactly half the length of the rotor. The $-2 \mathrm{mg} / \mathrm{s}$ streamline of Nakayama and Usui is quite similar to our $-2.75 \mathrm{mg} / \mathrm{s}$ streamline, with both extending a little farther axially than the $20 \mathrm{mg} / \mathrm{s}$ streamlines. We believe that this is excellent agreement.

Figures 2 and 3 present the $\theta$ and $v$ contours for Case $B$. In Case $A$, the contours of the region $0<r<0.7$ are a simple extrapolation of the region $0.7<$ $r<0.8$ shown in the figures; that is, the variations in $\theta$ and $v$ are confined within the neighborhoods of the endcaps. Both functions are high at the bottom endcap, low at the top endcap, and are odd functions about the midplane. 


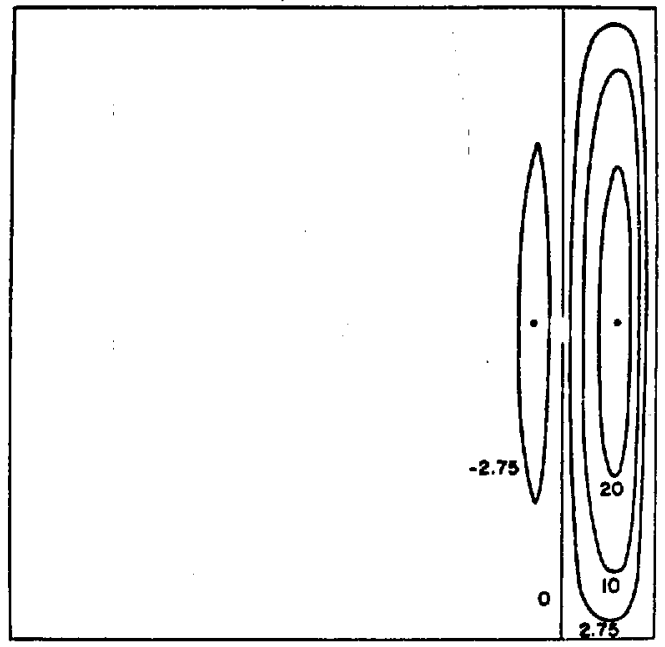

Fig. 1. Streamlines for the thermally driven $S_{6}$ centrifuge of Nakayama and Usui. ${ }^{5}$ The figure covers the region $0.7 \leqslant r \leqslant 1.0$ and $0 \leqslant z \leqslant 2 L$. Each streamline is labeled with the mass current in $\mathrm{mg} / \mathrm{s}$ between it and the dividing streamline labeied 0.

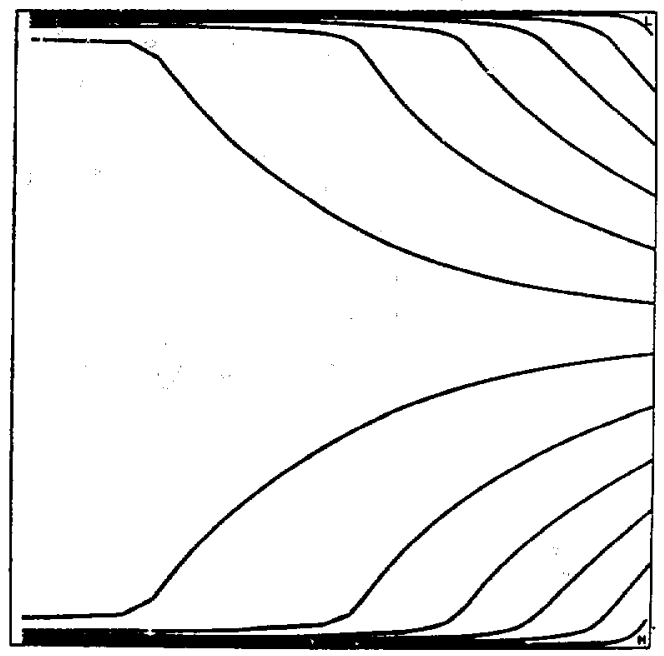

Pig. 2. Isotherms for the same region as covered in Fig. 1. The extremes are $\theta= \pm 1.56 \times 10^{-2}$, which is $\pm 5 K_{0}$. The contour spacina is $0.833 \mathrm{~K}$, and the las contour has a value of $315.42 \mathrm{~K} . \theta=0$ at the rotor midplane. 


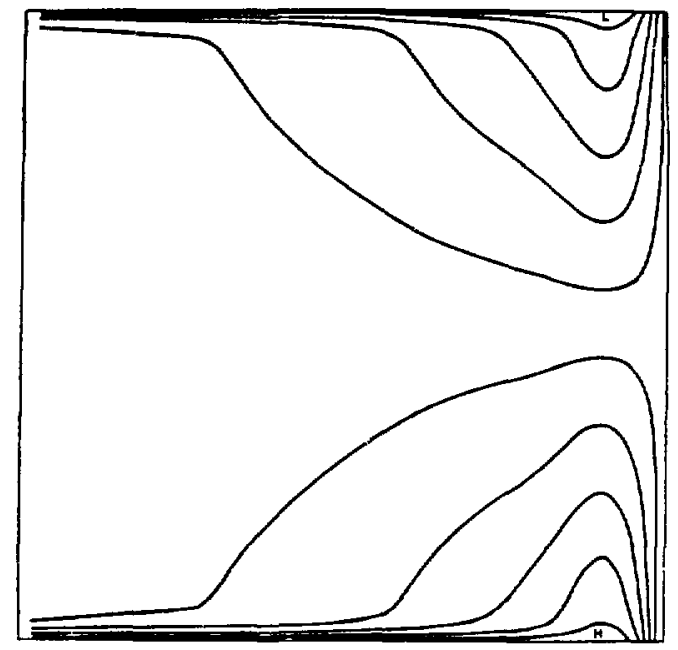

Fig. 3. Contours of $v$ for the same region as shown in Fig. 1. The extremes are $v= \pm 8.04 \times 10^{-3}$, which is $2.09 \mathrm{~m} / \mathrm{s}$. The contour snacing is 0.174 $\mathrm{m} / \mathrm{s}$ and the lowest contour shown has a value of $-0.784 \mathrm{~m} / \mathrm{s}$. Two of, the 12 contours, as shown in Fig. ?, cannot be distinguished hecause they lie on ton of the upper and zower graph boundaries. $v=0$ at the rotor midplane.

Figure 4 presents the heat flux through the bottom endcap as a function of $r$. The solid curve is from our calculation and the dashed line is from the analytic solution by Carrier and Maslen ${ }^{6}$ using our Case A numerical solution just outside the Ekman layers as boundary conditions on the analytic Ekman layer solution. The qualitative agreement is good, with almost no error near the peak. The relative error becomes large near $r=0.5$, although the absolute error is not too large.

The second example is an attempt to reproduce the mass driven solution shown in Fig. 6 of Nakayama and Usui. The parameters are the same as in the first example, except for the introduction of a net throughput rate of $10 n \mathrm{mg} / \mathrm{s}$. Feed is introduced at $r=0.5$ on the upper endcap. Material is removed from the rotor at $r=0.98$ on the upper endcap and $r=0.5$ on the bottom endcap. The cut is 0.5 . The grid used is the same as for the Case A thermally driven solution. The most striking feature of this solution is the narrow region of downflow between the ports at $r=0.5$ on both endcaps and the equally narrow region of upflow next to the rotor wall that feeds the exit port in the upper endcap. The grid is sufficiently refined next to the rotor wall to resolve the flow there. However, the downflow at $r=0.5$ is only one cell wide, so the resolution is too crufle to provide a meaningful comparison with the analytic solution. The radial zones need 


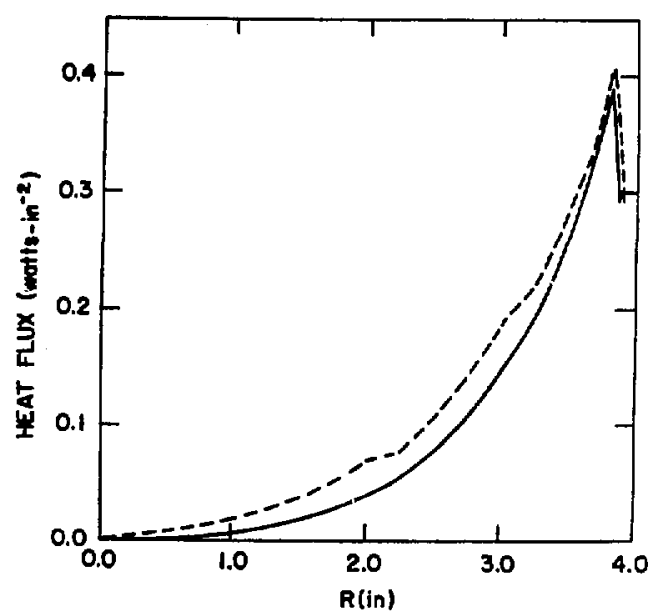

Fig. 4. Heat flux through the bottom enciap. The solid curve is our Case A numerical solution and the dashed line is an analytic solution.

to be narrower in this region, perhaps at the expense of resolution at smaller radii.

In addition to the zoning problem, this seemingly innocuous example contains a hidden disaster: it is decidedly nonlinear. First, the fluid just outside the stream at $r=0.5$, flowing from the top endcap to the bottom endcap, is not rotating in the laboratory frame $(v \simeq-1)$. In the same region, $\theta \approx 0.1$ and $p \approx$ 1.5. Near the axis, $p$ becomes as large as 2.1. It drops to -0.8 at the rotor wall. Nakayama and Usui argue that linear analysis may still be useful, but we believe that a more reliable approach would be to adjust our radial grid as discussed previously and rerun the problem with our nonlinear program.

\section{REFERENCES}

1. H. G. Wood and G. Sanders, "Rotating Compressible Flows with Internal Sources and Sinks," Union Carbide Corporation Nuclear Division report K/TS-10,250 (October 1980).

2. J. E. Park, "Calculation of the Isotope Distribution in a Gas Centrifuge," Union Carbide Corporation Nuclear Division report K/CSD/TM-36 (February 1981).

3. L. D. Cloutman, "A Novel Approach to the Computation of Axisymmetric Countercurrent Patterns in Gas Centrifuges," Los Alamos Scientific Laboratory report LA-8258-MS (February 1980). 
4. D. E. Arnurius, "Solving Large Systems of Linear Equations by Factorization," Union Carbide Corporation Nuclear Division report CSD-15 (1975).

5. W. Nakayama and S. Usui, "Flow in Rotating Cylinder of a Gas Centrifuge," J. Nucl. Sci. Technol. 11, 12 (1974).

6. G. F. Carrier, "Phenomena in Rotating Fluids," in Proceedings of the Eleventh International Congress of Applied Mechanics, Munich, Germany, 1964. 